\title{
Gas detectors for x-ray lasers
}

K. Tiedtke, ${ }^{1}$ J. Feldhaus, ${ }^{1}$ U. Hahn, ${ }^{1}$ U. Jastrow, ${ }^{1}$ T. Nunez, ${ }^{1}$ T. Tschentscher, ${ }^{1}$

S. V. Bobashev, ${ }^{2}$ A. A. Sorokin, $\left.{ }^{2, a}\right)$ J. B. Hastings, ${ }^{3}$ S. Möller ${ }^{3}$ L. Cibik, ${ }^{4}$ A. Gottwald, ${ }^{4}$

A. Hoehl, ${ }^{4}$ U. Kroth, ${ }^{4}$ M. Krumrey, ${ }^{4}$ H. Schöppe, ${ }^{4}$ G. Ulm, ${ }^{4}$ and M. Richter ${ }^{4, b)}$

${ }^{1}$ Deutsches Elektronen-Synchrotron, Notkestraße 85, 22603 Hamburg, Germany

${ }^{2}$ Ioffe Physico-Technical Institute, Polytekhnicheskaya 26, 194021 St. Petersburg, Russia

${ }^{3}$ Stanford Linear Accelerator Center, Stanford, California 94309, USA

${ }^{4}$ Physikalisch-Technische Bundesanstalt, Abbestraße 2-12, 10587 Berlin, Germany

(Received 22 January 2008; accepted 28 February 2008; published online 8 May 2008)

We have developed different types of photodetectors that are based on the photoionization of a gas at a low target density. The almost transparent devices were optimized and tested for online photon diagnostics at current and future x-ray free-electron laser facilities on a shot-to-shot basis with a temporal resolution of better than $100 \mathrm{~ns}$. Characterization and calibration measurements were performed in the laboratory of the Physikalisch-Technische Bundesanstalt at the electron storage ring BESSY II in Berlin. As a result, measurement uncertainties of better than $10 \%$ for the photon-pulse energy and below $20 \mu \mathrm{m}$ for the photon-beam position were achieved at the Free-electron LASer in Hamburg (FLASH). An upgrade for the detection of hard x-rays was tested at the Sub-Picosecond Photon Source in Stanford. (C) 2008 American Institute of Physics. [DOI: $10.1063 / 1.2913328$ ]

\section{INTRODUCTION}

For more than 100 years, $\mathrm{x}$-rays have been used to discover the structure of matter in many different disciplines (see, e.g., Ref. 1 and references therein). With the recent progress in development of free-electron lasers (FELs) providing femtosecond $\mathrm{x}$-ray pulses of high intensity, even the dynamics of chemical reactions and fast processes in materials may be studied in the near future. ${ }^{2-4} \mathrm{X}$-ray lasers also allow us to extend the investigation of strong-field phenomena on photon matter interaction from the optical region to the regime of the classical photoeffect above ionization thresholds. ${ }^{5-11}$ However, for many of these totally new experiments, a reliable online photon diagnostics is mandatory. In particular, the inherent statistical intensity fluctuations of FELs based on self-amplified spontaneous emission (SASE) require a pulse energy analysis on a shot-to-shot basis. A photodetector has been developed for this purpose that is based on the photoionization of a (rare) gas and is, thus, radiation hard. ${ }^{12}$ In contrast to classical ionization chambers ${ }^{13}$ the new detector is operated at a considerably lower gas pressure. Hence, it is almost transparent and, due to the suppression of secondary ionization processes in the gas and electron instead of ion detection, considerably faster. Moreover, in the vacuum-ultraviolet and soft x-ray regimes, the device is sensitive enough to be absolutely calibrated by using dispersed synchrotron radiation at a low photon intensity. The present work describes operation of those gasmonitor detectors (GMDs) as permanent parts of the online photon diagnostics at the soft $\mathrm{x}$-ray Free-electron LASer in Hamburg (FLASH). Moreover, we present extensions of the detector principle toward the determination of the FEL beam

\footnotetext{
a) Also at Physikalisch-Technische Bundesanstalt, Abbestraße 2-12, 10587 Berlin, Germany.

${ }^{b)}$ Electronic mail: mathias.richter@ptb.de.
}

position and the measurement of hard FEL x-rays at future facilities in Hamburg (European XFEL) and Stanford (Linac Coherent Light Source). First test results obtained at the SubPicosecond Photon Source (SPPS) in Stanford demonstrate that our GMD for hard $\mathrm{x}$-rays (X-GMD) represents a useful alternative to $\mathrm{X}$-ray detectors based on scattering and fluorescence. ${ }^{14,15}$

\section{GMDs AT FLASH}

Figure 1 shows the schematic of a GMD as currently used at FLASH. Similar to the former version, ${ }^{12}$ electrons and ions generated by photoionization of a target gas at a pressure of around $10^{-3} \mathrm{~Pa}$ are extracted by an almost homogeneous static electric field and detected by simple electrodes. Both charge signals can be read out in the pulse mode and the ion signal can also be read in the current mode with

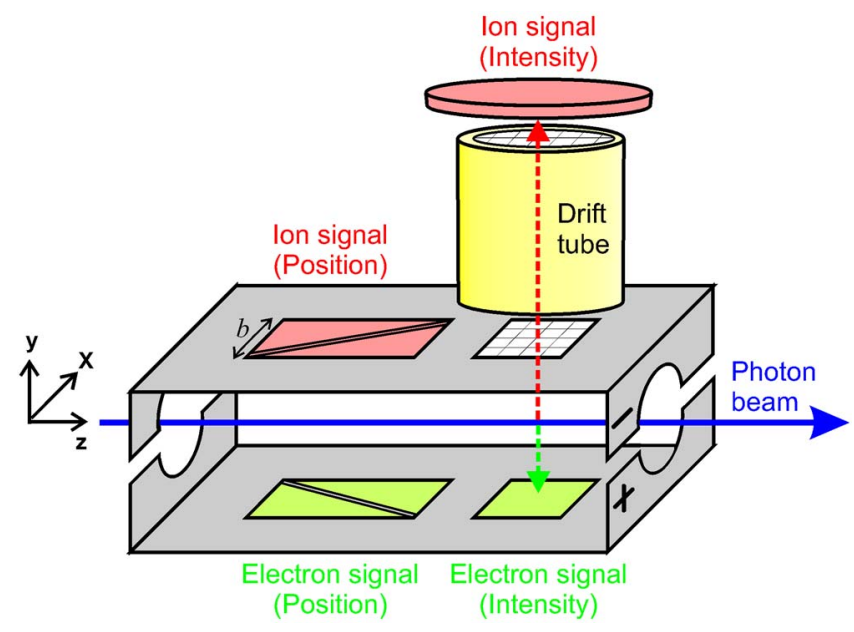

FIG. 1. (Color online) Schematic of a FLASH dual GMD for the online measurement of the photon intensity and the beam position. 
a time constant of $20 \mathrm{~s}$. The device was calibrated in the ion current mode at the laboratory of the PhysikalischTechnische Bundesanstalt (PTB), which is Germany's national metrology institute, at the electron storage ring BESSY II in Berlin, by using dispersed synchrotron radiation in the microwatt regime and a calibrated photodiode as a standard detector. $^{16,17}$ The resulting spectral responsivity $s_{\text {ion }}(\hbar \omega)$, which is defined as the ratio between the signal current $I_{\text {ion }}$ and the radiant power $\Phi$, may be expressed, at given photon energy $\hbar \omega$, by the quantum efficiency $Q E_{\text {ion }}(\hbar \omega)$ of the detector and the mean charge $q(\hbar \omega)$ generated per absorbed photon

$$
\begin{aligned}
s_{\text {ion }}(\hbar \omega) & =\frac{I_{\text {ion }}}{\Phi}=\frac{q(\hbar \omega) Q E_{\text {ion }}(\hbar \omega)}{\hbar \omega} \\
& =\frac{q(\hbar \omega) \sigma(\hbar \omega) z_{\text {ion }} \eta_{\text {ion }} n}{\hbar \omega}=\frac{q(\hbar \omega) \sigma(\hbar \omega) z_{\text {ion }} \eta_{\text {ion }} p}{\hbar \omega k T} .
\end{aligned}
$$

The acceptance length $z_{\text {ion }}$ along the photon beam for ion collection and the ion detection efficiency $\eta_{\text {ion }}$ are the true calibration quantities because the other quantities are known from literature (Boltzmann constant $k=1.381 \times 10^{-23} \mathrm{~J} \mathrm{~K}^{-1}$, $q(\hbar \omega)$ and the total photoionization cross section $\sigma(\hbar \omega)$ for the respective target (rare) gas as listed in Table I or accurately determined during the measurements (target gas pressure $p$ by using a calibrated spinning rotor vacuum gauge, temperature $T$ by means of a calibrated Pt100 resistance thermometer and, as a result, target particle density $n=p / k T$ ). The recommended $q$ and $\sigma$ values listed in Table I represent a new evaluation and compilation, with respect to the lowest uncertainty, of the experimental data given in Refs. 18-28. In Table II, the $z_{\text {ion }} \times \eta_{\text {ion }}$ values for the four GMDs currently operating at FLASH are listed and were measured with a relative standard uncertainty of $3.5 \%$. Thus, the spectral responsivities $s_{\text {ion }}$ of the GMDs may be calculated according to Eq. (1) with a relative standard uncertainty of better than $7 \%$.

The GMDs may also be used as ion time-of-flight mass/ charge spectrometers in the pulse mode. The drift tube in the ion path (see Fig. 1) allows this feature to be used for additional photon diagnostics such as higher FEL harmonics that lead to more highly charged ions. The pulse mode for electron detection, on the other hand, is used for fast quantitative FEL pulse energy measurements with a $30 \mathrm{~ns}$ temporal resolution. The electron pulse signal is calibrated by online comparison to the calibrated ion current and averaging over many FEL shots

$$
s_{\mathrm{el}}(\hbar \omega)=s_{\text {ion }}(\hbar \omega) \frac{\left\langle Q_{\mathrm{el}}\right\rangle}{\left\langle I_{\text {ion }}\right\rangle} \nu,
$$

where $Q_{\mathrm{el}}$ and $s_{\mathrm{el}}$ denote the charge detected per FEL pulse and the spectral responsivity for the electron pulse mode, respectively, and $v$ is the number of FEL pulses per time unit. This cross-calibration procedure finally results in a relative standard uncertainty for the measurement of photonpulse energy on the order of $10 \%$, which is dominated by the inherent statistical shot-to-shot fluctuations of a SASE-FEL like FLASH. The latter is apparent in Fig. 2, which shows the diagnostics panel in the FLASH control system with information about the FEL pulse energy measured by means of GMD 2.

In addition to FEL intensity measurements, each FLASH GMD also allows us to determine the photon beam position. Split electrodes are used for ion and electron detection, as shown in Fig. $1 .^{29-31}$ Due to the homogeneous extraction field, the charged particles created within a single photon pulse represent, in the plane of the respective split electrode, a projection of the photon beam. The lengths $z_{1}$ and $z_{2}$ of this projection on each part of a split electrode define the corresponding signal ratio according to

$$
\frac{I_{1}}{I_{2}}=\frac{z_{1}}{z_{2}}=\frac{b-2 \Delta x}{b+2 \Delta x} .
$$

$\Delta x$ and $b$ denote the deviation of the beam position from the center and the size of the split electrode in the respective direction perpendicular to the photon beam, as shown in Fig. 1. Equation (3) has been experimentally confirmed in the PTB laboratory at BESSY II by moving the GMDs through the temporally and spatially stable synchrotron radiation beam. For the ion current split electrode of GMD 2, the corresponding result is shown in Fig. 3. At FLASH, the two pairs of two perpendicularly oriented GMDs allow the determination of the horizontal and the vertical beam position at two different locations along the photon beam. Since the uncertainty in the beam position measurement amounts to about $20 \mu \mathrm{m}$ and the two locations along the photon beam are separated by $15 \mathrm{~m}$, the horizontal and the vertical beam direction can be determined online with an uncertainty in the microradiant regime by means of the four FLASH GMDs.

\section{GMDs FOR HARD X-RAY FELS}

With increasing photon energy, the photoabsorption and the photoionization cross sections of matter generally decrease. Even for xenon, the heaviest rare gas suited to be used as a target in a gas detector, the photoionization cross section at $10 \mathrm{keV}$ photon energy amounts to only about 4 $\times 10^{-20} \mathrm{~cm}^{2}=40 \mathrm{~kb}$ (Ref. 32) and is almost three orders of magnitude less than at $100 \mathrm{eV}$ (see Table I). This has to be taken into consideration when extending the GMD principle from the soft to the hard x-ray regime. The classical ionization chamber ${ }^{13}$ is one solution of a gas detector with a sufficient sensitivity in the hard x-ray regime. However, the high pressure needed (above $1 \mathrm{~Pa}$ ) would lead to space charge effects and secondary ionization processes within the gas that would then considerably reduce the temporal resolution of the detector (see, e.g., Fig. 2 in Ref. 33). Thus, for pulse-resolved measurements at FELs, we chose, as an alternative, the solution of working with a low target gas pressure but with an open electron multiplier as a signal amplifier for ion detection.

A schematic of our X-GMD suited for operation at target gas pressures below $0.1 \mathrm{~Pa}$ is shown in Fig. 4. The detector was designed to be tested at the SPPS in Stanford, ${ }^{34}$ which was, until its shutdown in 2006, a worldwide unique femtosecond $\mathrm{x}$-ray source with a rather moderate photon number per pulse, on the order of $10^{6}$ at the photon energy of 9.4 
TABLE I. Total photoionization cross section $\sigma$ and mean charge $q$ created per absorbed photon as a function of photon energy $\hbar \omega$ for the rare gases helium to xenon. The values represent a compilation of the experimental data given in Refs. $18-28 . e=1.602 \times 10^{-19} \mathrm{C}$ denotes the elementary charge. The spectral regions of strong resonances are omitted, respectively. Relative standard uncertainties are below $5 \%$ for the argon cross section, below $3 \%$ for all other cross sections, and below $2 \%$ for the $q$ values.

\begin{tabular}{|c|c|c|c|c|c|c|c|c|c|c|}
\hline \multirow[b]{2}{*}{$\begin{array}{c}\hbar \omega \\
(\mathrm{eV})\end{array}$} & \multicolumn{2}{|l|}{ Helium } & \multicolumn{2}{|c|}{ Neon } & \multicolumn{2}{|l|}{ Argon } & \multicolumn{2}{|l|}{ Krypton } & \multicolumn{2}{|c|}{ Xenon } \\
\hline & $\begin{array}{c}\sigma \\
(\mathrm{Mb})\end{array}$ & $q / e$ & $\begin{array}{c}\sigma \\
(\mathrm{Mb})\end{array}$ & $q / e$ & $\begin{array}{c}\sigma \\
(\mathrm{Mb})\end{array}$ & $q / e$ & $\begin{array}{c}\sigma \\
(\mathrm{Mb})\end{array}$ & $q / e$ & $\begin{array}{c}\sigma \\
(\mathrm{Mb})\end{array}$ & $q / e$ \\
\hline 14.0 & & & & & & & & & 64.42 & 1.00 \\
\hline 15.0 & & & & & & & 45.11 & 1.00 & 61.01 & 1.00 \\
\hline 16.0 & & & & & 29.90 & 1.00 & 45.66 & 1.00 & 56.43 & 1.00 \\
\hline 17.0 & & & & & 33.15 & 1.00 & 45.04 & 1.00 & 50.92 & 1.00 \\
\hline 18.0 & & & & & 35.15 & 1.00 & 43.95 & 1.00 & 45.80 & 1.00 \\
\hline 19.0 & & & & & 36.25 & 1.00 & 42.58 & 1.00 & 40.81 & 1.00 \\
\hline 20.0 & & & & & 36.70 & 1.00 & 40.86 & 1.00 & Resonances & Resonances \\
\hline 21.0 & & & & & 36.70 & 1.00 & 38.78 & 1.00 & Resonances & Resonances \\
\hline 22.0 & & & 6.60 & 1.00 & 36.17 & 1.00 & 36.65 & 1.00 & Resonances & Resonances \\
\hline 23.0 & & & 7.17 & 1.00 & 35.35 & 1.00 & 34.44 & 1.00 & Resonances & Resonances \\
\hline 24.0 & & & 7.69 & 1.00 & 34.19 & 1.00 & 32.20 & 1.00 & 20.35 & 1.00 \\
\hline 25.0 & 7.21 & 1.00 & 7.96 & 1.00 & 32.78 & 1.00 & Resonances & & 17.69 & 1.00 \\
\hline 26.0 & 6.79 & 1.00 & 8.20 & 1.00 & 31.12 & 1.00 & Resonances & & 15.35 & 1.00 \\
\hline 27.0 & 6.40 & 1.00 & 8.41 & 1.00 & Resonances & & Resonances & & 13.12 & 1.00 \\
\hline 28.0 & 6.05 & 1.00 & 8.59 & 1.00 & Resonances & & Resonances & & 11.19 & 1.00 \\
\hline 29.0 & 5.70 & 1.00 & 8.70 & 1.00 & Resonances & & 19.68 & 1.00 & 9.56 & 1.00 \\
\hline 30.0 & 5.38 & 1.00 & 8.80 & 1.00 & 23.23 & 1.00 & 17.33 & 1.00 & 8.08 & 1.00 \\
\hline 31.0 & 5.10 & 1.00 & 8.85 & 1.00 & 21.00 & 1.00 & 15.18 & 1.00 & 6.82 & 1.00 \\
\hline 32.0 & 4.82 & 1.00 & 8.89 & 1.00 & 18.59 & 1.00 & 13.33 & 1.00 & 5.80 & 1.00 \\
\hline 33.0 & 4.57 & 1.00 & 8.85 & 1.00 & 16.20 & 1.00 & 11.69 & 1.00 & 5.01 & 1.00 \\
\hline 34.0 & 4.32 & 1.00 & 8.80 & 1.00 & 13.83 & 1.00 & 10.24 & 1.00 & 4.34 & 1.03 \\
\hline 35.0 & 4.09 & 1.00 & 8.75 & 1.00 & 11.37 & 1.00 & 8.93 & 1.00 & 3.77 & 1.06 \\
\hline 36.0 & 3.88 & 1.00 & 8.70 & 1.00 & 8.89 & 1.00 & 7.75 & 1.00 & 3.29 & 1.09 \\
\hline 37.0 & 3.68 & 1.00 & 8.65 & 1.00 & 6.95 & 1.00 & 6.73 & 1.00 & 2.92 & 1.11 \\
\hline 38.0 & 3.50 & 1.00 & 8.59 & 1.00 & 5.55 & 1.00 & 5.86 & 1.00 & 2.60 & 1.14 \\
\hline 39.0 & 3.32 & 1.00 & 8.50 & 1.00 & 4.37 & 1.00 & 5.10 & 1.00 & 2.33 & 1.16 \\
\hline 40.0 & 3.16 & 1.00 & 8.40 & 1.00 & 3.41 & 1.00 & 4.44 & 1.01 & 2.12 & 1.18 \\
\hline 41.0 & 3.01 & 1.00 & 8.35 & 1.00 & 2.14 & 1.00 & 3.88 & 1.02 & 1.96 & 1.21 \\
\hline 42.0 & 2.86 & 1.00 & 8.30 & 1.00 & 1.71 & 1.00 & 3.41 & 1.03 & 1.83 & 1.22 \\
\hline 43.0 & 2.72 & 1.00 & 8.20 & 1.00 & 1.35 & 1.00 & 3.02 & 1.03 & 1.71 & 1.24 \\
\hline 44.0 & 2.60 & 1.00 & 8.10 & 1.00 & 1.11 & 1.01 & 2.68 & 1.04 & 1.62 & 1.26 \\
\hline 45.0 & 2.48 & 1.00 & Resonat & & 0.96 & 1.03 & 2.38 & 1.05 & 1.54 & 1.27 \\
\hline 46.0 & 2.38 & 1.00 & Resonal & & 0.83 & 1.04 & 2.12 & 1.05 & 1.47 & 1.28 \\
\hline 47.0 & 2.28 & 1.00 & Resonal & & 0.75 & 1.04 & 1.89 & 1.05 & 1.42 & 1.29 \\
\hline 48.0 & 2.19 & 1.00 & Resonal & & 0.72 & 1.05 & 1.68 & 1.06 & 1.37 & 1.31 \\
\hline 49.0 & 2.10 & 1.00 & Resonances & Resonances & 0.73 & 1.06 & 1.50 & 1.07 & 1.34 & 1.32 \\
\hline 50.0 & 2.02 & 1.00 & 7.50 & 1.00 & 0.74 & 1.07 & 1.34 & 1.08 & 1.31 & 1.33 \\
\hline 52.0 & 1.85 & 1.00 & 7.30 & 1.00 & 0.79 & 1.10 & 1.11 & 1.09 & 1.27 & 1.32 \\
\hline 54.0 & 1.71 & 1.00 & 7.10 & 1.00 & 0.88 & 1.11 & 0.95 & 1.10 & 1.24 & 1.31 \\
\hline 56.0 & 1.63 & 1.00 & 6.99 & 1.00 & 0.98 & 1.12 & 0.84 & 1.11 & 1.23 & 1.30 \\
\hline 58.0 & 1.58 & 1.00 & 6.81 & 1.00 & 1.05 & 1.13 & 0.75 & 1.12 & 1.23 & 1.30 \\
\hline 60.0 & Resonances & & 6.69 & 1.00 & 1.13 & 1.14 & 0.69 & 1.13 & 1.24 & 1.30 \\
\hline 62.0 & Resonances & & 6.57 & 1.00 & 1.17 & 1.15 & 0.64 & 1.14 & 1.26 & 1.32 \\
\hline 64.0 & Resonances & & 6.42 & 1.00 & 1.25 & 1.15 & 0.60 & 1.14 & 1.55 & 1.40 \\
\hline 66.0 & 1.15 & 1.00 & 6.29 & 1.00 & 1.30 & 1.16 & 0.58 & 1.15 & Resonar & \\
\hline 68.0 & 1.06 & 1.00 & 6.14 & 1.01 & 1.33 & 1.17 & 0.56 & 1.17 & Resonar & \\
\hline 70.0 & 0.98 & 1.00 & 6.00 & 1.01 & 1.36 & 1.17 & 0.54 & 1.17 & 3.06 & 1.79 \\
\hline 72.0 & 0.91 & 1.00 & 5.85 & 1.01 & 1.38 & 1.17 & 0.53 & 1.17 & 4.40 & 1.88 \\
\hline 74.0 & 0.84 & 1.00 & 5.69 & 1.02 & 1.39 & 1.17 & 0.52 & 1.17 & 6.14 & 1.94 \\
\hline 76.0 & 0.79 & 1.00 & 5.54 & 1.02 & 1.41 & 1.17 & 0.52 & 1.17 & 7.90 & 1.99 \\
\hline 78.0 & 0.74 & 1.00 & 5.38 & 1.03 & 1.42 & 1.17 & 0.52 & 1.17 & 9.90 & 2.03 \\
\hline 80.0 & 0.69 & 1.00 & 5.24 & 1.03 & 1.42 & 1.17 & 0.52 & 1.17 & 12.02 & 2.05 \\
\hline 82.0 & 0.65 & 1.00 & 5.08 & 1.03 & 1.41 & 1.18 & 0.52 & 1.17 & 14.02 & 2.08 \\
\hline 84.0 & 0.61 & 1.00 & 4.93 & 1.04 & 1.40 & 1.18 & 0.52 & 1.17 & 16.16 & 2.11 \\
\hline 86.0 & 0.58 & 1.01 & 4.78 & 1.04 & 1.39 & 1.18 & 0.52 & 1.18 & 18.99 & 2.13 \\
\hline 88.0 & 0.55 & 1.01 & 4.64 & 1.04 & 1.38 & 1.18 & 0.53 & 1.18 & 21.55 & 2.15 \\
\hline 90.0 & 0.52 & 1.01 & 4.50 & 1.04 & 1.36 & 1.18 & 0.54 & 1.27 & 23.50 & 2.16 \\
\hline 92.0 & 0.49 & 1.02 & 4.37 & 1.04 & 1.34 & 1.18 & Resonances & & 24.99 & 2.18 \\
\hline
\end{tabular}


TABLE I. (Continued.)

\begin{tabular}{|c|c|c|c|c|c|c|c|c|c|c|}
\hline \multirow[b]{2}{*}{$\begin{array}{c}\hbar \omega \\
(\mathrm{eV})\end{array}$} & \multicolumn{2}{|c|}{ Helium } & \multicolumn{2}{|c|}{ Neon } & \multicolumn{2}{|l|}{ Argon } & \multicolumn{2}{|l|}{ Krypton } & \multicolumn{2}{|c|}{ Xenon } \\
\hline & $\begin{array}{c}\sigma \\
(\mathrm{Mb})\end{array}$ & $q / e$ & $\begin{array}{c}\sigma \\
(\mathrm{Mb})\end{array}$ & $q / e$ & $\begin{array}{c}\sigma \\
(\mathrm{Mb})\end{array}$ & $q / e$ & $\begin{array}{c}\sigma \\
(\mathrm{Mb})\end{array}$ & $q / e$ & $\begin{array}{c}\sigma \\
(\mathrm{Mb})\end{array}$ & $q / e$ \\
\hline 94.0 & 0.46 & 1.02 & 4.24 & 1.05 & 1.32 & 1.18 & Resonances & & 26.24 & 2.19 \\
\hline 96.0 & 0.44 & 1.02 & 4.11 & 1.05 & 1.31 & 1.18 & Resonances & & 27.28 & 2.20 \\
\hline 98.0 & 0.42 & 1.02 & 3.99 & 1.06 & 1.29 & 1.18 & 1.26 & 1.89 & 27.91 & 2.21 \\
\hline 100.0 & 0.39 & 1.02 & 3.87 & 1.06 & 1.27 & 1.18 & 1.30 & 1.91 & 28.16 & 2.22 \\
\hline 102.0 & & & 3.75 & 1.06 & 1.26 & 1.18 & 1.36 & 1.91 & 27.95 & 2.23 \\
\hline 104.0 & & & 3.64 & 1.06 & 1.25 & 1.18 & 1.46 & 1.92 & 27.27 & 2.24 \\
\hline 106.0 & & & 3.53 & 1.07 & 1.23 & 1.18 & 1.59 & 1.94 & 26.30 & 2.24 \\
\hline 108.0 & & & 3.42 & 1.07 & 1.20 & 1.18 & 1.74 & 1.98 & 24.98 & 2.25 \\
\hline 110.0 & & & 3.32 & 1.07 & 1.18 & 1.18 & 1.87 & 2.03 & 23.31 & 2.25 \\
\hline 112.0 & & & 3.21 & 1.07 & 1.16 & 1.18 & 2.00 & 2.05 & 21.59 & 2.25 \\
\hline 114.0 & & & 3.11 & 1.07 & 1.14 & 1.18 & 2.16 & 2.08 & 19.80 & 2.26 \\
\hline 116.0 & & & 3.02 & 1.08 & 1.12 & 1.18 & 2.31 & 2.10 & 17.94 & 2.26 \\
\hline 118.0 & & & 2.93 & 1.08 & 1.10 & 1.18 & 2.47 & 2.13 & 16.13 & 2.27 \\
\hline 120.0 & & & 2.84 & 1.08 & 1.09 & 1.18 & 2.63 & 2.15 & 14.39 & 2.27 \\
\hline 124.0 & & & 2.67 & 1.08 & 1.06 & 1.18 & 2.95 & 2.19 & 11.18 & 2.28 \\
\hline 128.0 & & & 2.51 & 1.09 & 1.01 & 1.19 & 3.27 & 2.22 & 8.54 & 2.29 \\
\hline 132.0 & & & 2.36 & 1.09 & 0.96 & 1.19 & 3.56 & 2.24 & 6.53 & 2.29 \\
\hline 136.0 & & & 2.22 & 1.10 & 0.92 & 1.19 & 3.83 & 2.26 & 5.02 & 2.30 \\
\hline 140.0 & & & 2.09 & 1.10 & 0.89 & 1.19 & 4.04 & 2.27 & 3.86 & 2.31 \\
\hline 144.0 & & & 1.98 & 1.10 & 0.85 & 1.19 & 4.23 & 2.29 & 3.03 & 2.43 \\
\hline 148.0 & & & 1.87 & 1.11 & 0.81 & 1.19 & 4.43 & 2.30 & 2.33 & 2.53 \\
\hline 152.0 & & & 1.77 & 1.11 & 0.78 & 1.19 & 4.60 & 2.30 & 1.89 & 2.59 \\
\hline 156.0 & & & 1.67 & 1.11 & 0.74 & 1.19 & 4.74 & 2.32 & 1.59 & 2.66 \\
\hline 160.0 & & & 1.59 & 1.11 & 0.72 & 1.19 & 4.85 & 2.33 & 1.36 & 2.72 \\
\hline 164.0 & & & 1.50 & 1.11 & 0.69 & 1.20 & 4.92 & 2.33 & 1.18 & 2.77 \\
\hline 168.0 & & & 1.42 & 1.12 & 0.67 & 1.20 & 4.97 & 2.34 & 1.05 & 2.81 \\
\hline 172.0 & & & 1.35 & 1.12 & 0.64 & 1.20 & 5.01 & 2.34 & 0.97 & 2.83 \\
\hline 176.0 & & & 1.29 & 1.12 & 0.62 & 1.20 & 5.03 & 2.35 & 0.91 & 2.85 \\
\hline 180.0 & & & 1.22 & 1.12 & 0.60 & 1.20 & 5.04 & 2.36 & 0.88 & 2.87 \\
\hline 184.0 & & & 1.17 & 1.13 & 0.58 & 1.21 & 5.04 & 2.37 & 0.88 & 2.85 \\
\hline 188.0 & & & 1.11 & 1.13 & 0.56 & 1.21 & 5.05 & 2.37 & 0.89 & 2.84 \\
\hline 192.0 & & & 1.06 & 1.13 & 0.54 & 1.21 & 5.05 & 2.37 & 0.92 & 2.83 \\
\hline 196.0 & & & 1.01 & 1.14 & 0.52 & 1.21 & 5.06 & 2.38 & 0.96 & 2.82 \\
\hline 200.0 & & & 0.97 & 1.14 & 0.51 & 1.22 & 5.06 & 2.38 & 1.00 & 2.81 \\
\hline 204.0 & & & 0.92 & 1.14 & 0.49 & 1.22 & 5.06 & 2.40 & 1.05 & 2.80 \\
\hline 208.0 & & & 0.88 & 1.14 & 0.47 & 1.22 & 5.07 & 2.43 & 1.10 & 2.78 \\
\hline 212.0 & & & 0.84 & 1.14 & 0.45 & 1.22 & Resonances & & 1.14 & 2.77 \\
\hline 216.0 & & & 0.81 & 1.14 & 0.44 & 1.23 & Resonances & & 1.19 & 2.77 \\
\hline 220.0 & & & 0.77 & 1.14 & 0.42 & 1.23 & Resonances & & 1.24 & 2.78 \\
\hline 224.0 & & & 0.74 & 1.14 & 0.41 & 1.23 & 5.28 & 2.49 & 1.28 & 2.79 \\
\hline 228.0 & & & 0.71 & 1.15 & 0.39 & 1.23 & 5.25 & 2.49 & 1.33 & 2.80 \\
\hline 232.0 & & & 0.69 & 1.15 & 0.37 & 1.23 & 5.22 & 2.49 & 1.37 & 2.81 \\
\hline 236.0 & & & 0.66 & 1.15 & 0.36 & 1.23 & 5.18 & 2.49 & 1.41 & 2.81 \\
\hline 240.0 & & & 0.63 & 1.15 & 0.34 & 1.24 & 5.13 & 2.50 & 1.44 & 2.82 \\
\hline 244.0 & & & 0.61 & 1.15 & 0.32 & 1.24 & 5.08 & 2.51 & 1.47 & 2.82 \\
\hline 248.0 & & & 0.58 & 1.15 & Resonances & & 5.02 & 2.52 & 1.50 & 2.82 \\
\hline 252.0 & & & 0.56 & 1.15 & Resonances & & 4.96 & 2.53 & 1.52 & 2.82 \\
\hline 256.0 & & & 0.54 & 1.15 & 1.15 & 1.49 & 4.89 & 2.54 & 1.53 & 2.82 \\
\hline 260.0 & & & 0.52 & 1.15 & 1.54 & 1.60 & 4.82 & 2.55 & 1.54 & 2.82 \\
\hline 264.0 & & & 0.50 & 1.15 & 1.93 & 1.72 & 4.75 & 2.55 & 1.55 & 2.82 \\
\hline 268.0 & & & 0.48 & 1.15 & 2.32 & 1.83 & 4.67 & 2.56 & 1.56 & 2.83 \\
\hline 272.0 & & & 0.47 & 1.15 & 2.71 & 1.95 & 4.60 & 2.57 & 1.57 & 2.83 \\
\hline 276.0 & & & 0.45 & 1.16 & 3.05 & 2.05 & 4.52 & 2.58 & 1.58 & 2.84 \\
\hline 280.0 & & & 0.43 & 1.16 & 3.00 & 2.06 & 4.45 & 2.59 & 1.58 & 2.84 \\
\hline 284.0 & & & 0.42 & 1.16 & 2.96 & 2.08 & 4.38 & 2.62 & 1.59 & 2.84 \\
\hline 288.0 & & & 0.40 & 1.16 & 2.92 & 2.10 & 4.31 & 2.65 & 1.59 & 2.84 \\
\hline 292.0 & & & 0.39 & 1.17 & 2.88 & 2.12 & 4.25 & 2.66 & 1.58 & 2.85 \\
\hline 296.0 & & & 0.38 & 1.17 & 2.84 & 2.13 & 4.18 & 2.67 & 1.58 & 2.85 \\
\hline 300.0 & & & 0.37 & 1.17 & 2.80 & 2.14 & 4.12 & 2.67 & 1.58 & 2.85 \\
\hline
\end{tabular}


TABLE II. $z_{\text {ion }} \times \eta_{\text {ion }}$ values as the relevant calibration quantities of the four GMDs currently operating at FLASH obtained at two different ion extraction fields (200 and $400 \mathrm{~V} / \mathrm{cm})$. For a given photon energy $\hbar \omega$, a total photoionization cross section $\sigma$, and a mean charge $q$ created per absorbed photon according to Table I and a temperature $T$ and a target gas pressure $p$, the spectral responsivities $s_{\text {ion }}$ of the detectors may be calculated according to Eq. (1) with a relative standard uncertainty of better than $7 \%$, respectively.

\begin{tabular}{ccc}
\hline \hline & \multicolumn{2}{c}{$\begin{array}{c}z_{\text {ion }} \times \eta_{\text {ion }} \\
(\mathrm{mm})\end{array}$} \\
GMD & $200 \mathrm{~V} / \mathrm{cm}$ & $400 \mathrm{~V} / \mathrm{cm}$ \\
\hline 1 & $26.0 \pm 0.9$ & $26.6 \pm 0.9$ \\
2 & $25.8 \pm 0.9$ & $26.2 \pm 0.9$ \\
3 & $26.9 \pm 0.9$ & $28.7 \pm 1.0$ \\
4 & $27.0 \pm 0.9$ & $28.9 \pm 1.0$ \\
\hline \hline
\end{tabular}

$\mathrm{keV}$. To further increase the detector sensitivity, the X-GMD has the option to collect photoions created over the comparably large distance of $20 \mathrm{~cm}$ along the photon beam by means of an additional extraction field maintained by an aperture system (see Fig. 4). Since in the hard x-ray regime, the collection of the highly energetic electrons would require collection and extraction voltages of tens of $\mathrm{keV}$, the $\mathrm{X}-\mathrm{GMD}$ is restricted to ion detection only. The open multiplier with an open area of $1.5 \times 1.5 \mathrm{~cm}^{2}$ and a maximum gain of $3 \times 10^{6}$ consists of $25 \mathrm{Al}_{2} \mathrm{O}_{3}$ dynodes and was optimized and, with a pulsed electron gun, tested for high linearity recording from 1 to $10^{6}$ charged particles per pulse without any saturation. The ionization chamber is vacuum sealed by beryllium windows at the photon beam's entrance and exit.

Figure 5 shows the spectral responsivity curves of the
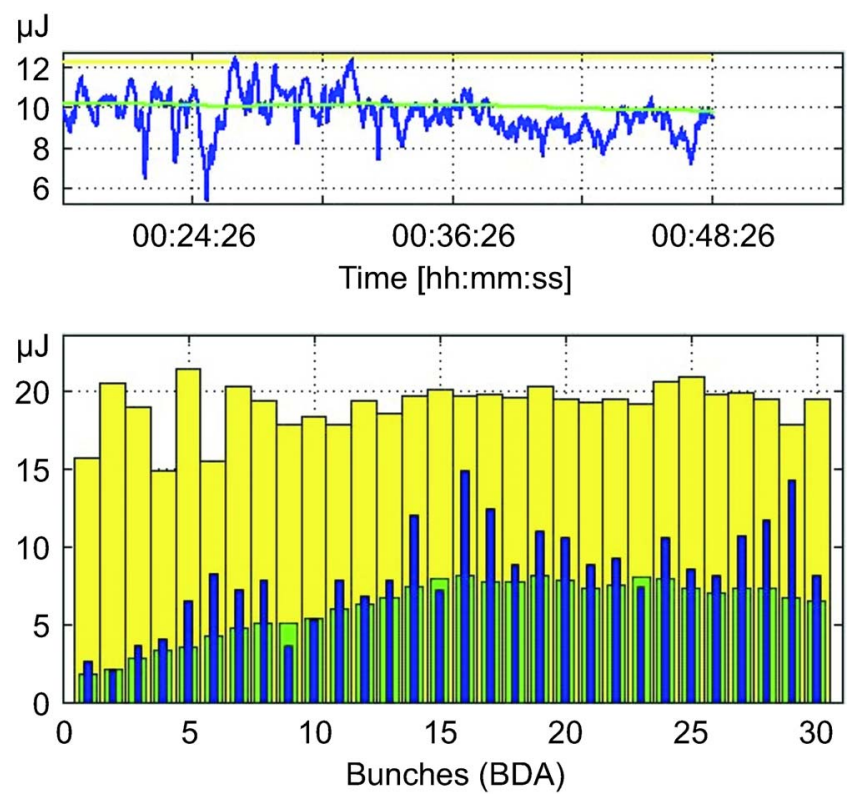

FIG. 2. (Color online) Diagnostics panel in the FLASH control system showing the GMD 2 intensity signals. The upper window shows the mean pulse energy taken from the ion-current signal, whereas the lower window shows the pulse energies of the individual pulse trains measured via the electron signal on a shot-to-shot basis (narrow bars for current values, thick bars for maximum values since the panel was turned on, and medium bars for averaged values since the panel was turned on).

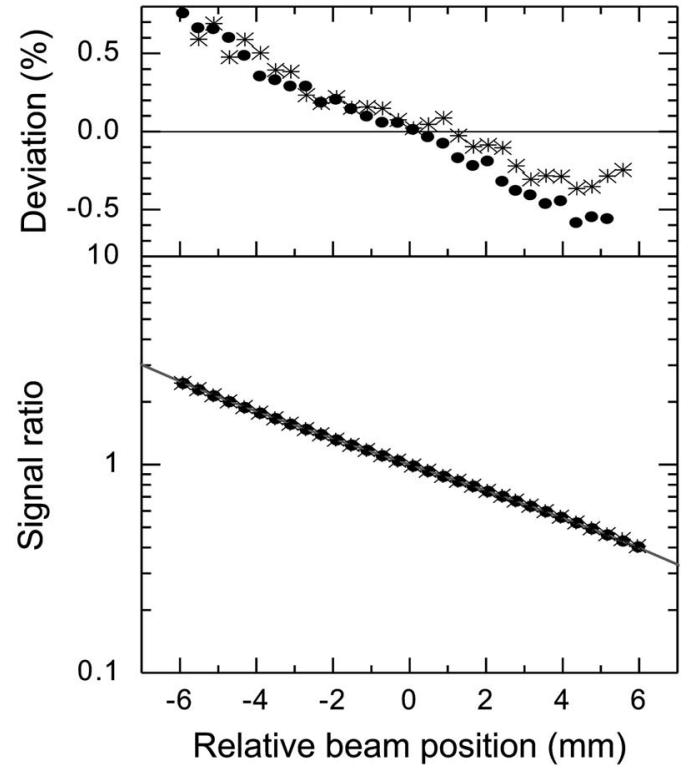

FIG. 3. Signal ratio for the ion current split electrode of GMD 2 as a function of the relative beam position (bottom). The symbols show two independently measured data sets and the solid line is a result calculated according to Eq. (3). At the top part, the deviation of the theoretical curve from the experimental data is depicted.

X-GMD for xenon with a multiplier voltage of $U_{\mathrm{MP}}=$ $-3.5 \mathrm{kV}$, i.e., a multiplier gain of $3 \times 10^{6}$, normalized to the target particle density, for photon energies from 4 to $10 \mathrm{keV}$ with and without additional ion collection along the photon beam. The data have been obtained with a relative standard uncertainty of $5 \%$ at the four crystal X-ray monochromator beamline of PTB at BESSY II. ${ }^{35}$ We compared the X-GMD signal current measured with a Keithley 617 picoamperemeter to the output of a calibrated semiconductor photodiode as secondary standard for radiant power, traceable to a cryogenic radiometer as the primary detector standard. ${ }^{17}$ As expected and indicated by the prominent $L$ edges around $5 \mathrm{keV}$, the calibration curves clearly follow the xenon photoionization cross section [see Eq. (1)]. Moreover, at the photon energy of $9.4 \mathrm{keV}$, the quantum efficiency $Q E$ with additional ion collection along the photon beam can be estimated to be about $3 \times 10^{-6}$ for a xenon target gas pressure of $0.1 \mathrm{~Pa}$.

However, when testing the X-GMD at the SPPS, the

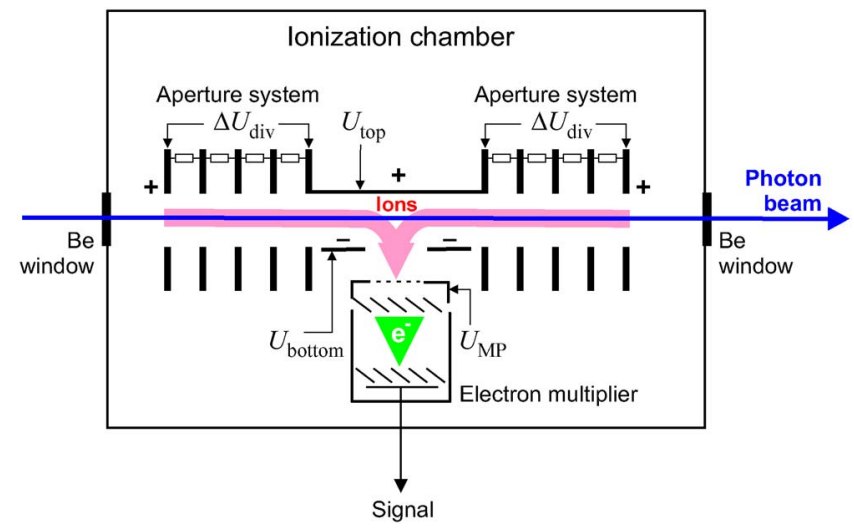

FIG. 4. (Color online) Schematic of the X-GMD within its ionization chamber. 


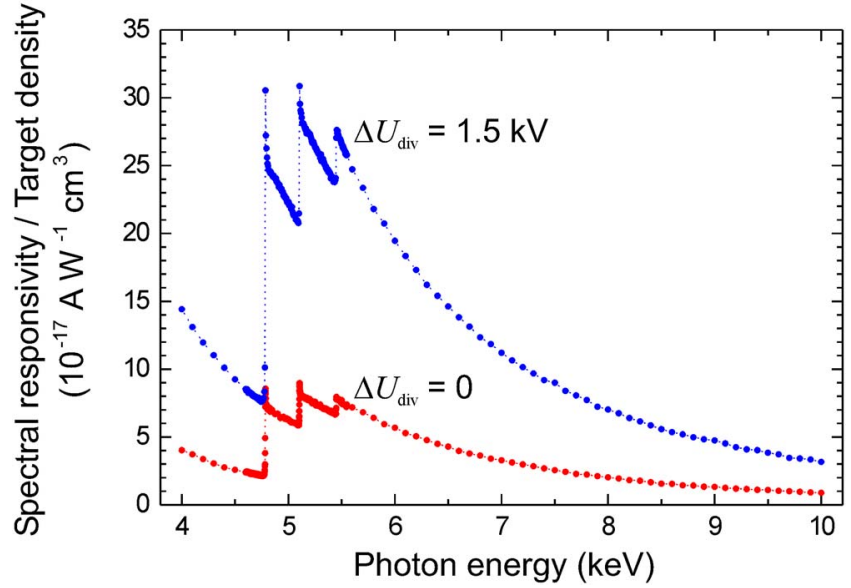

FIG. 5. (Color online) Spectral responsivity of the X-GMD at $3.5 \mathrm{kV}$ multiplier voltage, normalized to the xenon target particle density, with $\left(\Delta U_{\text {div }}\right.$ $=1.5 \mathrm{kV})$ and without $\left(\Delta U_{\mathrm{div}}=0\right)$ additional ion collection along the photon beam.

number of photoions detected per pulse was in the order of 1 , i.e., the photon number per pulse was found to be $10^{6}$ or less. Therefore, in order to determine the SPPS output, accumulation over hundreds of $\mathrm{x}$-ray pulses was realized to measure the average pulse intensity by means of a digital storage
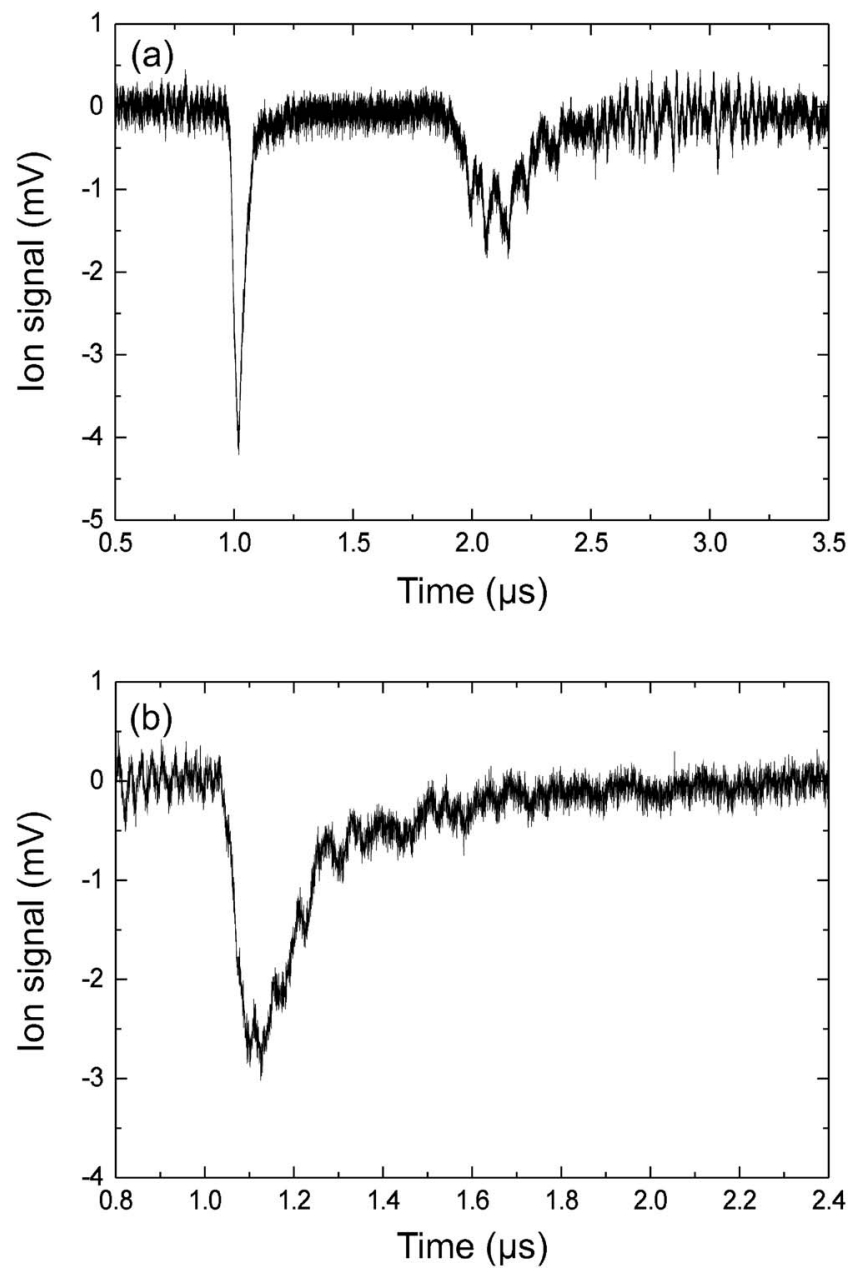

FIG. 6. Photoion signal of the X-GMD with (a) and without (b) additional ion collection along the photon beam as measured at the SPPS in January 2006.

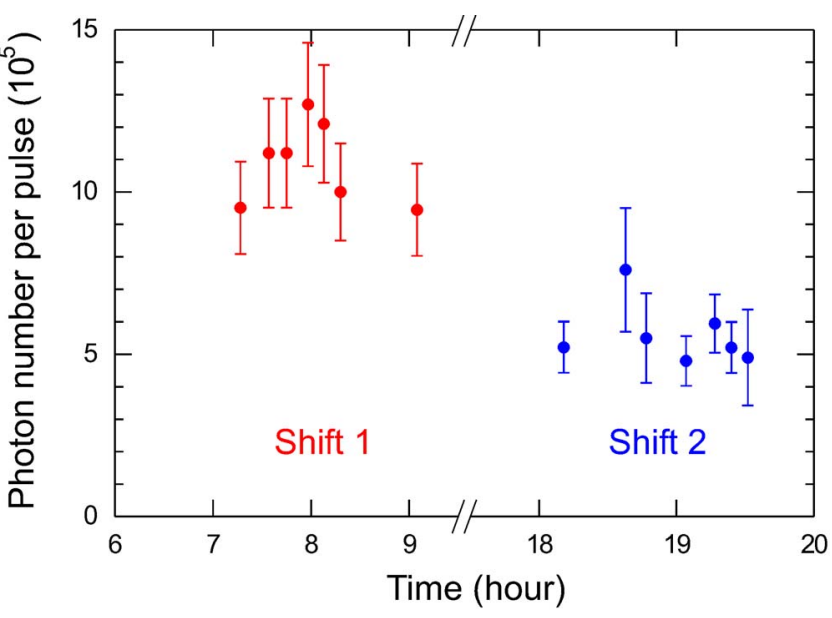

FIG. 7. (Color online) Mean photon number per pulse as measured in January 2006 at the SPPS.

oscilloscope. Figure 6(a) shows an example of the pulseresolved photoion signal of the X-GMD, averaged over 2126 SPPS photon pulses, which yields an average of $5 \times 10^{5}$ photons per SPPS pulse. The sharper peak around $1 \mu$ s (full width at half maximum $<100 \mathrm{~ns}$ ) corresponds to ions collected from the inner detector region near the multiplier, whereas signals arising between 1.8 and $2.5 \mu$ s are due to ions collected from the outer region within the aperture system along the photon beam (Fig. 4). The latter vanish when turning off the corresponding divider voltage $\Delta U_{\text {div }}$. The obvious fine structure is explained by the charge distribution and, hence, a corresponding time-of-flight distribution of the photoions. ${ }^{36}$ A corresponding structure was also observed at the first peak of photoions generated within the inner region of the GMD. This structure is demonstrated by Fig. 6(b), which shows the X-GMD signal with the divider voltage $\Delta U_{\text {div }}$ turned off. Figure 7 shows the result of an evaluation of the mean photon number per pulse as measured during two shifts at the SPPS in January 2006.

\section{SUMMARY}

In summary, we have demonstrated that photodetectors based on the photoionization of (rare) gases and detection of the charged photofragments represent versatile tools for the online measurement of pulse energies on a shot-to-shot basis at current and future x-ray FEL facilities. At FLASH, we have even extended the principle of our so-called GMDs to the online determination of the beam position. Moreover, to compensate for the lower photoionization cross sections in the hard X-ray regime, an upgrade (X-GMD) has been presented with signal amplification up to $10^{6}$ by means of an open electron multiplier for ion detection. Our gas detectors have a temporal resolution of better than $100 \mathrm{~ns}$. Measurement uncertainties of below $10 \%$ for the pulse energy and about $20 \mu \mathrm{m}$ for the beam position may be achieved. Pulseto-pulse instabilities are dominated by signal statistics and less than $1 \%$ for pulses of more than $10^{10}$ photons.

\footnotetext{
${ }^{1}$ A. Haase, G. Landwehr, and E. Umbach, Röntgen Centennial: X-Rays in Natural and Life Sciences (World Scientific, Singapore, 1997).

${ }^{2}$ V. Ayvazyan et al., Phys. Rev. Lett. 88, 104802 (2002).
} 
${ }^{3}$ J. Feldhaus, J. Arthur, and J. B. Hastings, J. Phys. B 38, S799 (2005).

${ }^{4}$ W. Ackermann et al., Nat. Photonics 1, 336 (2007).

${ }^{5} \mathrm{H}$. Wabnitz et al., Nature (London) 420, 482 (2002).

${ }^{6} \mathrm{H}$. Wabnitz et al., Phys. Rev. Lett. 94, 023001 (2005).

${ }^{7}$ A. A. Sorokin, S. V. Bobashev, K. Tiedtke, and M. Richter, J. Phys. B 39 , L299 (2006).

${ }^{8}$ A. A. Sorokin, S. V. Bobashev, K. Tiedtke, M. Wellhöfer, and M. Richter, Phys. Rev. A 75, 051402(R) (2007).

${ }^{9}$ M. Nagasono et al., Phys. Rev. A 75, 051406(R) (2007).

${ }^{10}$ R. Moshammer et al., Phys. Rev. Lett. 98, 203001 (2007).

${ }^{11}$ A. A. Sorokin, S. V. Bobashev, T. Feigl, K. Tiedtke, H. Wabnitz, and M. Richter, Phys. Rev. Lett. 99, 213002 (2007).

${ }^{12}$ M. Richter et al., Appl. Phys. Lett. 83, 2970 (2003).

${ }^{13}$ J. A. R. Samson, J. Opt. Soc. Am. 54, 6 (1964).

${ }^{14}$ R. Alkire, G. Rosenbaum, and G. Evans, J. Synchrotron Radiat. 7, 61 (2000).

${ }^{15}$ E. M. Dufresne, J. A. Guzman, S. B. Dierker, R. Clarke, D. A. Arms, and D. A. Walko, AIP Conf. Proc. 705, 679 (2004).

${ }^{16}$ R. Klein, M. Krumrey, M. Richter, F. Scholze, R. Thornagel, and G. Ulm, Synchrotron Radiat. News 15, 23 (2002).

${ }^{17}$ A. Gottwald, U. Kroth, M. Krumrey, M. Richter, F. Scholze, and G. Ulm, Metrologia 43, S125 (2006).

${ }^{18}$ D. R. Denne, J. Phys. D 3, 1392 (1970).

${ }^{19}$ W. S. Watson, J. Phys. B 5, 2292 (1972).

${ }^{20}$ G. V. Marr and J. B. West, At. Data Nucl. Data Tables 18, 497 (1976).

${ }^{21}$ B. X. Yang and J. Kirz, Appl. Opt. 26, 3823 (1987).

${ }^{22}$ J. A. R. Samson, L. Lyn, G. N. Haddad, and G. C. Angel, J. Phys. IV 1,
C99 (1991).

${ }^{23}$ I. H. Suzuki and N. Saito, ETL Tech. Rep. 56, 688 (1992), in Japanese.

${ }^{24}$ N. Saito and I. H. Suzuki, Int. J. Mass Spectrom. Ion Process. 115, 157 (1992).

${ }^{25}$ A. R. Samson, Z. X. He, L. Yin, and G. N. Haddad, J. Phys. B 21, 887 (1994).

${ }^{26}$ M. Bizau and F. J. Wuilleumier, J. Electron Spectrosc. Relat. Phenom. 71, 205 (1995).

${ }^{27}$ A. A. Sorokin, L. A. Shmaenok, S. V. Bobashev, B. Möbus, and G. Ulm, Phys. Rev. A 58, 2900 (1998).

${ }^{28}$ M. Richter, G. Ulm, Ch. Gerth, K. Tiedtke, J. Feldhaus, A. A. Sorokin, L. A. Shmaenok, and S. V. Bobashev, AIP Conf. Proc. 652, 165 (2003).

${ }^{29}$ A. Gottwald, S. V. Bobashev, U. Hahn, A. Hoehl, U. Jastrow, M. Richter, A. A. Sorokin, and K. Tiedtke, Proc. SPIE 5534, 13 (2004).

${ }^{30}$ K. Sato, T. Kudo, M. Suzuki, and T. Ishikawa, Spring-8 Annual Report, 1998 (unpublished), p. 181.

${ }^{31}$ A. Koyama, S. Sasaki, and T. Ishikawa, Rev. Sci. Instrum. 60, 1953 (1989).

${ }^{32}$ A. L. Henke, E. M. Gullikson, and J. C. Davis, At. Data Nucl. Data Tables 54, 181 (1993).

${ }^{33}$ A. A. Sorokin et al., Appl. Phys. Lett. 89, 221114 (2006).

${ }^{34}$ L. Bentson et al., Nucl. Instrum. Methods Phys. Res. A 507, 205 (2003).

${ }^{35}$ M. Krumrey and G. Ulm, Nucl. Instrum. Methods Phys. Res. A 467-468, 1175 (2001).

${ }^{36}$ T. Tonuma, A. Yagishita, H. Shibata, T. Koizumi, T. Matsuo, K. Shimay, T. Mukoyama, and H. Tawara, J. Phys. B 20, L31 (1987). 